\title{
Students' proposed self-management strategies in response to written cases depicting situations of adversity
}

\author{
Jared Davidson ${ }^{1}$ (D), Claire Simmonds ${ }^{1}$, Karen Whitfield ${ }^{2}$ (D), Kyle John Wilby ${ }^{3}$ (D) \\ ${ }^{1}$ School of Pharmacy, University of Otago, Dunedin, New Zealand \\ 2 School of Pharmacy, University of Queensland, Woolloongabba, Australia \\ ${ }^{3}$ College of Pharmacy, Faculty of Health, Dalhousie University, Halifax, Canada
}

\author{
Keywords \\ Emotional intelligence \\ Emotional regulation \\ Resilience \\ Pharmacy \\ Correspondence \\ Kyle John Wilby \\ College of Pharmacy \\ Dalhousie University \\ 5968 College St \\ PO Box 15000 \\ Halifax, NS \\ Canada B3H 4R2 \\ kyle.wilby@dal.ca
}

\begin{abstract}
Introduction: Pharmacy students are facing academic and non-academic pressures that require emotional regulation. This study explored students' possible self-management strategies when encountering situations known to deplete resilience. Methods: This was a qualitative think-aloud study designed to elicit final year pharmacy students' reactions to situations known to deplete resilience and evoke emotional responses (racism, lack of trust, negative feedback, burnout, personal stress, sexual harassment). Thematic analysis was used to capture the strategies students used to self-manage their emotions. Results: Students made use of three types of processes to self-manage their emotions, which were used to construct three overarching strategies: the internalizer (avoidance, self-reflection), the seeker (asking for help or corroboration), and the confronter (approaching the situation and persons involved 'head on'). Conclusion: Findings support the notion that students' self-management is not a 'one size fits all' construct, and any approach to emotional skill development needs to recognize individualization within student responses.
\end{abstract}

\section{Introduction}

Health professional students are increasingly facing both academic and non-academic pressures that may elicit negative emotional responses (Heinen Bullinger \& Kocalevent, 2017; Ribeiro et al., 2018; Bexelius et al., 2019; McKerrow et al., 2020). These responses could, in turn, have detrimental impacts on wellbeing, mental health, and resilience. Examples of such situations may include high workload, burnout, receiving negative feedback, personal matters (family or financial pressure), poor grades or patient interactions on clinical placements, racism, discrimination, or others (Beal et al., 2015; Perry et al., 2016; Hill, Goicochea \& Merlo, 2018). Emotional responses may also be unpredictable and exaggerated during times of acute stress. Supporting students to better manage or regulate emotional responses when faced with difficult situations may, therefore, be a useful strategy to avoid the negative consequences of these increasing demands (Johnson, 2015).

The concept of emotional regulation is a core component of emotional intelligence (EI) (Goleman, 1995; Bradberry \& Greaves, 2009). El is the ability to monitor one's own and others' emotions, discriminate among them, and use the information to guide one's thinking and actions (Goleman, 1995). El can be divided into five branches: self-awareness, self-management, motivation, social awareness, and relationship management (Goleman, 1995; Bradberry \& Greaves, 2009). The branch of self-management describes how a person regulates their emotions when reacting to specific situations. Self-management involves selfreflection, thoughtfulness, integrity, and openness to change. For example, a person who is able to effectively regulate their emotions may suppress or redirect negative emotional responses by focusing on selfreflection and personal growth. On the other hand, a 
person who does not effectively self-manage their emotions may be prone to internal (e.g. stress and anxiety) and external (e.g. outbursts, arguments) consequences that may negatively impact wellbeing and resilience. Despite being influenced by many personal factors, self-management is a skill that can be practised and developed through training, reflection, and feedback (Shahid, Stirling \& Adams, 2018; Foster, Cuzzillo \& Furness, 2018).

Emotional intelligence and self-management have been studied in the context of health professions education. Findings from medical students within a surgical setting show that those deemed more emotionally intelligent experience higher stress but recover faster (Arora et al., 2011). Numerous studies have shown a positive link between emotional intelligence and academic performance (Chew, Zain \& Hassan, 2013; MacCann et al., 2020). Consequently, there are increasing calls for emotional skill development to be incorporated within health professional education programmes (Chew et al., 2013). An important factor is that emotional intelligence has been shown to deplete over time (Stratton, Saunders \& Elam, 2008). Although the literature provides a good overview of the association between emotional intelligence, student stress, and performance, there is currently a lack of understanding of self-management behaviours students exhibit when faced with adversity. It is also unknown if students modify their behavioural self-management strategies when exposed to situations of different contexts.

The literature investigating emotional regulation mostly shows that students tend not to face the situation head-on when asked to recall memorable events or dilemmas (Rees, Monrouxe \& McDonald, 2013; Doulougeri, Panagopoulou \& Montgomery, 2016). Avoiding conflict, engaging in distractions, suppressing emotions, and focusing on other tasks are all known to be strategies used by students to regulate their emotional responses. Responses can also have lingering effects as students move through coursework and the programme (Monrouxe et al., 2014). To date, the literature focuses mainly on students' recall of experiences and, therefore, could be biased towards these situations that elicit more negative and lasting impressions. Understanding how students react and manage their emotions 'in the moment' remains yet to be explored.

Given the increasing demands on health professional students and the negative implications of stress for both personal wellbeing and academic success, it is imperative that programmes provide students with the skills and support necessary to face adversity. The theoretical construct of self-management within emotional intelligence may be a key to unlocking new ways of approaching student training and needs. However, before such content can be developed, one must begin to understand how students react and manage their emotions. This study investigated how students process their reactions and regulation strategies when faced with situations known to deplete resilience.

\section{Methods}

This qualitative study was conducted using a thinkaloud procedure (Van Semeren, Barnard \& Sandberg, 1994), combined with a structured interview, to elicit students' reactions to situations known to deplete resilience and evoke negative emotional responses. By having participants 'think aloud', as opposed to relying on recall, think-aloud protocols aim to get as close as possible to determining the thought processes of participants when reviewing material or other stimulation. Thematic analysis was then used to interpret the data and capture the strategies students used to self-manage (Braun \& Clarke, 2006). Guidance was followed to support the rigour of the research procedures, as described below (Sutton \& Austin, 2015). The study was conducted in accordance with the Declaration of Helsinki with no potential harm to participants; the anonymity of participants was guaranteed, and informed consent was obtained prior to study procedures. Departmental approval to conduct this study was provided, and full ethical review was exempted.

The study was conducted at the School of Pharmacy, University of Otago. The School maintains a Bachelor of Pharmacy accredited by the Pharmacy Council of New Zealand. The programme graduates approximately 120-140 students per academic year. In addition to lectures and small group case-based learning sessions, all students complete frequent simulations within a professional skills laboratory and clinical placements in community, hospital, and rural practices.

A total of 20 final-year pharmacy students were recruited for participation based on previously published similar studies using the same methods (Greenhill et al., 2015). Students were convenience sampled from a population of 130 final-year students. Recruitment advertisements were posted to closed social media sites for eligible participants. Those interested were instructed to contact study investigators for more information. All final-year students had previous resilience training that occurred two months prior to the start of this study (Dresser, Whitfield et al., 2021). 
Six cases were developed (Table I) based on a literature review of situations known to deplete student resilience and evoke negative emotional reactions (racism, lack of trust, negative feedback, burnout, personal matters, sexual harassment). Each student was then scheduled for a meeting with one of the student investigators. After training the student investigators in interview techniques, it was decided that a senior investigator would not be present during the interviews so that participants would speak freely to their peers. After providing written informed consent, participants began the think-aloud interview procedure. The investigator asked participants to review each case aloud and then express their thoughts, feelings and reactions as they moved through the case. Investigators prompted them by saying 'please think aloud' if participants became silent or did not state any thoughts, feelings, or reactions. Once each case was fully read, investigators asked: 'How do you think [the person] is feeling?', 'How do you think you would feel in this situation?', and 'How would you react in this situation?'. All interviews were audiorecorded. Participants were able to skip any case they did not feel comfortable answering.

Table I: Case scenarios used to elicit students' thought processes during think-aloud protocol

\begin{tabular}{ll}
\hline Case scenarios & Stephen is a 35-year-old Asian male working in a hospital pharmacy setting. The manager of the hospital pharmacy, a \\
Racism & within the institution and the provision of services. On this particular Monday, the manager began talking about \\
budget cuts being forced on the department by the manager's boss, who also happens to be Asian. While explaining \\
the budget cuts, the manager refers to his boss using an accent that appears to mimic how his boss speaks English. \\
Stephen automatically feels uncomfortable, especially as he is the only Asian pharmacist in the room. His other \\
colleagues appear to glance at him but smile nervously.
\end{tabular}

Lack of Trust

Negative Feedback

Burnout

Personal Stress

Sexual Harassment
Fiona is a recent graduate from the School of Pharmacy and is currently completing her internship year in an urban pharmacy in Auckland. For the last three months, she has been at the same site and has not been 'allowed' to speak to patients. Her internship supervisor says she is 'not ready', although she was able to counsel and educate patients regularly during her P4 rotations. Despite asking for opportunities, she is constantly turned down and told 'maybe next week'. As her assessment centre is fast approaching, she is beginning to stress about her ability to practice her skills before challenging the exam.

Jeremy is a 21-year-old male in his final year of pharmacy who recently finished a mini-OSCE and is confident that he nailed it and will receive only praise from the tutor. Upon meeting the tutor, however, he received negative feedback about his performance. Specifically, the tutor said that he had a 'lack of empathy' which would have made the patient feel very uncomfortable. The tutor assigned Jeremy a score of 'needs improvement' and suggested he practices 'a lot' before challenging the final OSCE that he must pass to pass the program. Jeremy felt cheated and confused by this feedback and wonders how his perception could have been so different from that of the tutors.

Regina is a 19-year-old student who has just started her first year of pharmacy. She has been struggling to get used to the new workload, as it is much higher than her pre-pharmacy courses. Before pharmacy, Regina would always make sure every assignment was 'perfect' and would study very hard for each test. With the increasing workload of pharmacy, she is finding it harder and harder to put in the same effort and has noticed that her grades are not as good as they used to be. This drop in her grades has caused her to lose motivation and wonder if she is going to be able to even get through the program.

Matt is a 19-year-old male in his second year of pharmacy. He is happy as he has just finished one of his assignments and can begin to focus on studying for the upcoming MCQ exam. His Grandmother, who lives in Australia, has been unwell for a year now. His family have been communicating for the last few weeks that things were not looking good. While up late studying for his midterm test, he receives a message from his Dad. He is told his Mom is flying to Australia, as his Grandmother has just passed away. His parents think he should stay at university and continue to study rather than travel to Australia for the funeral. Matt is upset as he would like to say goodbye to his Grandmother but understands, leaving midway through the semester could interfere with his uni. Matt feels overwhelmed by grief and stress of the looming midterm exam.

Gretchen is a 24-year-old intern in a community pharmacy. She loves getting to know her patients and has built good relationships with them. Her preceptor, Aaron, has encouraged her to practice counselling sildenafil patients. She is confident at having these conversations with male patients and finds most are respectful. Gretchen is preparing to take out a repeat to one of the sildenafil patients she has not spoken with yet. When she takes out the repeat, the patient begins to tell her how much the medicine has improved his sex life, and he loves how he is able to last longer. Gretchen acknowledges that it is good that he is having a positive experience with the medication but feels very uncomfortable with his information sharing. The patient then proceeds to ask her how old she is and that he likes her uniform before leaving with his medication. 
Interview recordings were transcribed verbatim by one investigator and checked by another. Three transcripts were then inductively coded during a meeting with three investigators. Codes were assigned by identifying segments that related to the research question and reflected students' management strategies. One investigator then coded the remaining transcripts, and coding was checked by another. At this point, it was evident that a pattern was appearing with the strategies students were using to manage their reactions to each scenario. Investigators met and labelled each of these as categories and then recoded all data according to the categories. Results were discussed by research team members over multiple occasions until all agreed on the final interpretation of the data and agreement on each category. Representative quotes were extracted from the transcripts to illustrate each strategy identified. The proportions of students adopting each strategy were calculated per case.

\section{Results}

Twenty students (ten females and ten males) responded to the advertisements and completed the think-aloud protocol. A total of $45 \%$ of participants identified themselves as Asian ethnicity, 50\% New Zealand European, and 5\% as Maori. On average, the interview protocol lasted approximately 30 minutes. No student decided to skip any case, noting no adverse effects from the study protocol.

The final strategies identified through the qualitative analysis were: the internalizer, the seeker, and the confronter. Although there was inevitably some crossover between strategies, the authors deemed the actions and behaviours associated with each to be largely independent of the others. Students adopted different strategies depending on the case situation. Although some students exhibited dominant strategies, the nature of the cases appeared to influence which strategy was able to capture their self-regulation processes. Table II provides the proportion of students adopting each strategy for each case.

Table II: Proportion of students adopting self-management strategies per case

\begin{tabular}{|c|c|c|c|c|c|c|}
\hline Strategy & Scenario 1 & Scenario 2 & Scenario 3 & Scenario 4 & Scenario 5 & Scenario 6 \\
\hline The Internalizer & $9(45 \%)$ & $2(10 \%)$ & $6(30 \%)$ & $6(30 \%)$ & $9(45 \%)$ & $11(55 \%)$ \\
\hline The Seeker & $4(20 \%)$ & $4(20 \%)$ & $6(30 \%)$ & $14(70 \%)$ & $10(50 \%)$ & $8(40 \%)$ \\
\hline The Confronter & $7(35 \%)$ & $14(70 \%)$ & $8(40 \%)$ & $0(0 \%)$ & $1(5 \%)$ & $1(5 \%)$ \\
\hline
\end{tabular}

\section{Strategies}

\section{The Internaliser}

The internaliser self-manages independently and without the support of others. Some internalisers are reflective and will process their emotions through the lens of personal growth. Even if they disagree with the situation or outcome, they will use feedback constructively to focus on self-betterment.

'I would just try and step back and try and get a bigger picture of the whole situation.'

'I'd take the words because obviously, they're trying to help because they're more experienced than you so any feedback is good feedback.'

Other internalisers are non-reflective and may attempt to discredit or ignore the situation altogether. It is also possible that the specific situation or feedback provided did not simply resonate with these internalisers.

I'd probably try and brush it off and know people are weird, like not take it to heart'
Finally, some internalisers may manage emotional reactions by distancing themselves from others when faced with difficult situations. These internalisers avoid situations that make them feel uncomfortable in order to save face and to decrease the risk of re-exposure to the negative stimulus.

'I feel like personally, I actually wouldn't say much. Just because I would be scared on how to approach it and like if it would affect anything in the future'

'I'm like not big on challenging, I'm not going to be like tell me what I need to fix, I would just kinda like suffer in silence and be angry.'

\section{The Seeker}

The seeker is the person who asks for help from others. They will not choose to directly confront the person or situation but will instead first reach out to family members, friends, colleagues, supervisors, or programme administrators for support and guidance. Guidance requests may be general or specific. Seekers attempt to identify 'allies' before acting further. 
'I would've asked for help from family'

'I would probably go tell my preceptor about it. They're on your side, they don't want you to feel uncomfortable especially around patients'

Seekers may also attempt to confirm or disconfirm the appropriateness of their emotional reactions with others to validate their feelings and actions. They may want to make comparisons with their peers or seek advice on whether or not they are under or overreacting.

I could sorta ask what other people did, what feedback they got. Just to compare myself to other people and see if I am getting the same treatment as everyone else is, with like what we said.'

\section{The Confronter}

The confronter is the person who addresses a situation head-on. They are quick to question and provide their own point of view, whether or not it is asked for. They will explain their perspective and, at times, may challenge the viewpoint of the person they are reacting to. They seek feedback directly from the source rather than seeking another opinion or validation of their responses.

'Yeah, I'd be pretty angry with my supervisor. I'd like to talk to him about why I'm not ready and what I have to do to show him that I'm ready... I don't think I'd go behind him and look anywhere else. I'd look to be pretty upfront with him about how I'm feeling and how I can sort it out.'

'I'd try and confront the manager and say how you I thought it was inappropriate for them to be doing that'

Confronters are direct but may use tactics such as negotiation to achieve their intended outcomes. Negotiation may be about resolving the current situation or preventing future occurrences by using strategies such as compromise.

'I'd maybe say, "would you consider letting me talk to patients? Under your supervision of course." And maybe bring up that the supervisor could maybe come and sit in and speak up at any time if she feels like I'm not ready.'

'I'd probably try to hint to them and see like what they'd kinda do from that. Not be too forward and be like, "I need time". But just hint to them in terms of saying, "I feel comfortable doing that if I could have a go one time. Maybe could you supervise $m e^{\prime \prime}$.

\section{Discussion}

This study aimed to explore possible self-management strategies of final-year students when faced with situations known to deplete resilience in real-time. It revealed that students' self-management techniques could be categorised according to three strategies: the internaliser, the seeker, and the confronter. Although, at times, students adopted a dominant strategy, most cycled between them, depending on the context of the situation they encountered. Each constructed strategy provides insight into the behaviours students use to deal with stressful situations and, collectively, they provide a foundation for understanding student reactions in times of emotional regulation.

Interestingly, students adopted different strategies depending on the nature of the interaction they encountered. This finding was unexpected, as it was believed that students would be more consistent in how they approached difficult situations based on other literature (Rees et al., 2013; Doulougeri, 2016). This finding demonstrates that context likely has a considerable effect on the types of reactions and behaviours we can expect from students when attempting to manage their emotions. This finding is also essential for educators. When discussing potential actions students should take when faced with difficult situations, one often fails to encourage discussion and open dialogue with those involved (e.g. preceptor, colleague, tutor). This study's findings suggest that although this strategy (e.g. confrontation) may work for some students, others may prefer to manage their emotions in alternative ways (e.g. seek support, internalise feedback) before attacking the situation 'head-on'. This finding aligns with previous work from medical students' memorable dilemmas and incidents (Rees et al., 2013; Doulougeri et al., 2016). In one study, only a small percentage of students (13.2\%) reported taking obvious or direct action in the face of their selfidentified dilemma (Rees et al., 2013). In the other study, the most common reaction to a challenging situation was noted to be inaction through internalisation (Doulougeri et al., 2016). Combined, findings call for a better understanding of the process that students use to regulate their emotions, which will assist programmes in developing pathways and training for students during times of great risk (e.g. high stakes assessment, clinical placements). Supporting students to recognise these times of stress and effectively manage their behaviours in accordance with their own preferences should be goals for programmes moving forward.

Think-aloud was used to target how students would act in real-time, as opposed to allowing them time to think about what they 'should' do given the situation 
presented. Previous studies investigating emotional regulation mainly relied on student recall (Rees et al., 2013; Monrouxe et al., 2014; Doulougeri et al., 2016). This may account for the large number of students describing avoidance as their typical self-management strategy. This study's results provide a greater understanding of how students self-manage across a variety of situations that may elicit different levels of emotional responses. This study's results add to the literature by identifying that, along with avoidance, students also commonly seek help and confront the situation. The 'seeker', for example, is an important finding. As students appear to commonly seek support from others (family, friends, staff, colleagues), programmes should ensure students are aware of resources available to support them and reminded of these resources as they progress through the programme and its different components. Students completing clinical placements or elective work outside of the main university context may need further orientation on the support systems available for them if they require or prefer to seek help.

This study's findings have implications for practice and research. There is increasing interest in facilitating emotional skill development in health professional programmes. This study's findings suggest that selfmanagement strategies are individualised and differ between students. As there is likely not one 'gold standard' strategy for managing emotional responses, programming should be designed to increase awareness of the resources available to students and to provide them with examples of effective ways to confront, seek help/support, or internalise their emotions in response to negative stimuli. Future research should further investigate students' selfmanagement behaviours and attempt to understand how each strategy (e.g. internaliser, seeker, confronter) impacts student success and wellbeing. Instead of attempting to change student behaviour to be more confronting or seeking, efforts should be made to recognise how different self-management strategies resonate across students and situations and provide students with multiple pathways for effectively regulating their emotional responses. Future research should also explore the links (if any) between selfmanagement, emotional intelligence theory, and student wellbeing and resilience.

The results of this study should be interpreted in light of some limitations. First, a standard set of cases was used to elicit students' reactions. It is possible that some of these situations did not resonate with students and/or did not evoke an emotional response. This may have skewed results towards the internaliser, as these students would not have the need to confront or seek help. Secondly, some cultural and contextual factors might have modified the way students self-manage across different settings. This should be a point for future research. Finally, the strategies constructed may not be inclusive of all behaviours students use to selfmanage their emotions when faced with resiliencedepleting situations. The validity of these findings should, therefore, be assessed in future studies. Despite these limitations, this study's findings are important and provide a foundation for the development of emotional skill programming within health professionals education.

The ability of health professionals to effectively selfmanage their emotions is a skill that needs to be learned and practised. As such, training programmes have a prominent role in initiating this programming in undergraduate and graduate curricula. When faced with possible resilience-depleting situations, students primarily self-manage their emotions using one of three strategies: internalising, seeking help, or confronting. These findings support the notion that self-management is not a 'one size fits all' construct, and this study's approach to emotional skill development needs to recognise individualisation within student responses. Future research should aim to better understand the effectiveness of each approach to help students identify which selfmanagement strategies may best suit the context of their emotional response.

\section{References}

Arora, S., Russ, S., Petrides, K.V, et al. (2011). Emotional intelligence and stress in medical students performing surgical tasks. Acad Med, 86, 1311-1317. https://doi.org/10.1097/ACM.0b013e31822bd7aa

Beall, J.W., DeHart, R.M., Riggs, R.M., \& Hensley, J. (2015). Perceived Stress, Stressors, and Coping Mechanisms among Doctor of Pharmacy Students. Pharmacy (Basel) J, 3(4), 344354. https://doi.org/10.3390/pharmacy3040344

Bexelius, T., Lachmann, H., Jambert-Pettersson, H., Kalen, S., Moller, R., \& Ponzer, S. (2019). Stress among medical students during clinical courses: a longitudinal study using contextual activity sampling system. Int J Med Educ, 10, 6874. https://doi.org/10.5116/ijme.5c94.9391

Bradberry, T., \& Greaves, J. (2009). Emotional intelligence 2.0. San Diego: TalentSmart

Braun, V., \& Clarke, V. (2006). Using thematic analysis in psychology. Qual Res in Psychol., 3, 77-101. https://doi.org/10.1191/1478088706qp063oa

Chew, B.H., Zain, A., \& Hassan, F. (2013). Emotional intelligence and academic performance in first and final year medical students: a cross-sectional study. BMC Med Educ, 13, 44. https://doi.org/10.1186/1472-6920-13-44 
Doulougeri, K., Panagopoulou, E., \& Montgomery, A. (2016). (How) do medical students regulate their emotions? BMC Med Educ, 16, 312. https://doi.org/10.1186/s12909-0160832-9

Dresser, J.D., Whitfield, K.M., Kremer, L.J., \& Wilby, K.J. (2021). Exploring how postmillennial pharmacy students balance life priorities and avoid situations known to deplete resilience. Am J Pharm Educ, 85(4), 8369. https://doi.org/10.5688/ajpe8369

Foster, K., Cuzzillo, C., \& Furness, T. (2018). Strengthening mental health nurses' resilience through a workplace resilience programme: a qualitative inquiry. $J$ Psychiatr Ment Health Nurs, 25, 338-348.

https://doi.org/10.1111/jpm.12467

Goleman, D. (1995). Emotional Intelligence. New York: Bantam Books. https://doi.org/10.1037/e538982004-001

Greenhill, J., Fielke, K.R., Richards, J.N., Walker, L.J., \& Walters, L.K. (2015). Towards an understanding of medical resilience in longitudinal integrated clerkships. BMC Med Educ, 15, 137. https://doi.org/10.1186/s12909-015-0404-4

Heinen, I., Bullinger, M., \& Kocalevent, R. (2017). Perceived stress in first year medical students - associations with personal resources and emotional distress. BMC Med Educ, 17, 4. https://doi.org/10.1186/s12909-016-0841-8

Hill, M.R., Goicochea, S., \& Merlo, L.J. (2018). In their own words: stressors facing medical students in the millennial generation. Med Educ Online, 23, 1530558. https://doi.org/10.1080/10872981.2018.1530558

Johnson, D.R. (2015). Emotional intelligence as a crucial component to medical education. Int J Med Educ, 6, 179-83. https://doi.org/10.5116/ijme.5654.3044

MacCann, C., Jiang, Y., Brown, L.E.R., Double, K.S., Bucich, M., \& Minbashian, A. (2020). Emotional intelligence predicts academic performance: a meta-analysis. Psychol Bull, 146, 150-186. https://doi.org/10.1037/bul0000219

McKerrow, I., Carney, P.A., Caretta-Weyer, H., Furnari, M., \& Miller Juve, A. (2020). Trends in medical students' stress, physical, and emotional health throughout training. Med Educ Online, 25(1), 1709278.

https://doi.org/10.1080/10872981.2019.1709278

Monrouxe, L.V., Rees, C.E., Endacott, R., \& Ternan, E. (2014). "Even now it makes me angry": health care students' professionalism dilemma narratives. Med Educ, 48, 502517. https://doi.org/10.1111/medu.12377

Perry, S.P., Hardeman, R., Burke, S.E., Cunningham, B., Burgess, D.J., \& van Ryn, M. (2016). The impact of everyday discrimination and racial identity centrality on African American medical student wellbeing: a report from the medical student CHANGE study. J Racial and Ethnic Health Disparities. 3, 519-526. https://doi.org/10.1007/s40615015-0170-3

Rees, C.E., Monrouxe, L.V., \& McDonald, L.A. (2013). Narrative, emotion and action: analysing 'most memorable' professionalism dilemmas. Med Educ, 47, 80-96. https://doi.org/10.1111/j.1365-2923.2012.04302.x
Ribeiro, I., Pereira, R., Freire, I.V., de Oliveira, B.G., Casotti, C.A., \& Boery, E.N. (2018). Stress and quality of life among university students: a systematic literature review. Health Prof Educ, 4, 70-77.

https://doi.org/10.1016/j.hpe.2017.03.002

Shahid, R., Stirling, J., \& Adams, W. (2018). Promoting wellness and stress management in residents through emotional intelligence training. Adv Med Educ Pract, 20, 681-686. https://doi.org/10.2147/AMEP.S175299

Stratton, T.D., Saunders, J.A., \& Elam, C.L. (2008). Changes in medical students' emotional intelligence: an exploratory study. Teach Learn Med, 20, 279-284.

https://doi.org/10.1080/10401330802199625

Sutton, J., \& Austin, Z. (2015). Qualitative research: data collection, analysis, and management. Can J Hosp Pharm, 68, 226-231. https://doi.org/10.4212/cjhp.v68i3.1456

Van Semeren, M.W., Barnard, Y.F., (1994). Sandberg, J.A.C. The Think Aloud Method: A Practical Guide to Modelling Cognitive Processes. London: Academic Press 